the patient, under these circumstances, has only himself to blame for his lamentable condition. Will it not be otherwise, if the trade succeed in obtaining their charter?

\title{
Medical Students and Ophthalmology
}

It is little wonder that the vexed question of what the medical student should be taught of ophthalmology has aroused interest far from these shores. The Archives of Ophthalmology recently printed a weighty paper by Dr. Gordon M. Byers, of Montreal, giving his views upon the subject. Post-graduate instruction does not offer the same difficulties as pre-graduate instruction, mainly, perhaps, because it falls mainly to teachers in the specialities for solution, while under-graduate studies in any one subject are but a single factcr in a much larger problem, in which workers in all branches of medicine are interested. Nobody may at this day hope to master all medical knowledge during his studentship, whatever may have been the case once. The foremost place must be assigned to disorders that involve the issues of life and death, and then to those that lead to complete and permanent disablement. Lastly, come the disorders which are neither menacing nor rare, but of importance since they are common. Judged from this utilitarian standpoint how many eye problems fall into these several groups? Among the major questions are intraocular tumours, malignant growths on and about the eye, wounds and injuries of the orbit, orbital cellulitis, and osteitis from extension or metastasis; the student should be able to distinguish between the various disorders of which proptosis is the most striking sign. Diseases that are danger signals include optic neuritis, choked disc, retinitis or choroiditis, palsies of the muscles of the eve, and visual disturbances expressive of disease in the optic tract. - Disorders that threaten disablement include purulent ophthalmia, trachoma, diphtheria of the conjunctiva, wounds, injuries and burns of the eye. There is demanded a fair knowledge of refraction, ophthalmoscopy, and perimetry in the functional examination of the eye. Byers appears to think that by a re-arrangement of the present curriculum and by the introduction of more effective methods of teaching and study, room could be made for the necessary amount of ophthalmology, which could be given in four months' whole time work, provided the student had had a year of active work in general surgery. Finally, he raises a strong plea for the continued retention of ophthalmology in the general curriculum of medicine. It is clearly impossible that a man could intelligently practise in any special field without a knowledge of medicine and surgery as a whole. 